\title{
A NEW APPROACH TO THE ANALYSIS OF THE CONTACT SURFACES OF ROLLING KINEMATIC COUPLE
}

\author{
Libor Beneš ${ }^{1}$, Rudolf Kaloč ${ }^{2}$, Luboš Minář ${ }^{3}$ \\ 1, 2, ${ }^{3}$ Dept of Mechanics, Materials and Machine Parts, Jan Perner Transport Faculty, \\ University of Pardubice, Studentská 95, 53009 Pardubice, Czech Republic \\ E-mail: ${ }^{1}$ Libor.Benes@upce.cz (corresponding author)
}

Received 17 February 2009; accepted 15 November 2010

\begin{abstract}
All physical processes of contact phenomenon especially a slip mechanism occur in the superficial layers related to changes in the micro/macro-geometry of the surface. It is also the area where thermo-mechanical processes of non-isothermal nature arise. The persistent problems of damage to contact surfaces when being dynamically strained may only be gradually resolved using up-to-date metallographic methods, i.e. an experimental analysis of samples taken from the contact-stressed areas. This paper deals with a new theoretical approach to the loading analysis of contact surfaces during rolling and gives basic information about the study on the influence of dynamical loading in the contact area with the possible occurrence of wave effects in contact with the adhesion drive of a rail vehicle (as an example). The experimental part is based on laboratory testing applying a new-type designed device.
\end{abstract}

Keywords: contact surfaces, rolling kinematical pair, dynamical loading, slip (adhesion) characteristic, contact temperatures.

\section{Introduction}

The persistent problems of damage to contact surfaces when being dynamically strained may only be gradually resolved using up-to-date metallographic methods, i.e. an experimental analysis of samples taken from the contact-stressed areas. However, this generally known fact discloses its difficulties. The paper primarily focuses on the facts that result from the possible problems related to knowledge of loading modes.

In principle, there are two basic approaches. One is the analysis of samples taken from the contact area of a real component which has been subjected to work load. This method is generally used in cases of evaluating the state obviously showing the existing erosion of the contact surface.

However, preventing the occurrence of contact layer erosion requires such laboratory processes that can be based on knowledge about the nature of loading material samples and its history and, especially, on information characterizing the dynamics of loading. This is crucial (and still widely ignored) for roll-away type contact pairs. Special attention is paid to the wheel and rail contact pair which is particularly a typical example of dynamic loading. In spite of this, most of the analyzed forces are unfortunately based on the classical Hertz theory.
For example, the research by Beneš et al. (2008) points out the fact that the contact spot is the area where force effect is of impact nature. From the view of material, it is necessary to consider the influence of speed on deformation that can affect all material constants, including the basic parameters of fracture mechanics.

An additional phenomenon, which must be paid special attention, is the origination of contact temperatures, which, especially as a result of tangential forces, may reach values at which the structural influence of material may occur in the local areas (Beneš and Záhorová 2006).

\section{The Phenomenon of the Occurrence of Possible Wave Effects in the Contact Area}

The volumes of real material in the contact area constitute a continuum in which, under specific conditions of loading, energy is transferred by means of wave effects.

The impact nature of the load can be described by amplitude density with a band spectrum, which in reality can overlap the line spectrum of natural frequencies that is typical of the continuous environment of the contact areas.

Before analytical speculation based on the research by Brepta (1997), it is necessary to point out that the as- 
sumption of a homogenous continuum in the given case is not absolutely correct due to the existence of a considerably influenced thin superficial layer. Its dimension is very small in comparison with the dimensions of the contacting bodies. Therefore, the indicated speculation can be considered a useful guide towards the values of the occurring dynamic stresses.

The compression wave propagates in the direction of the normal line of contact $x$ at dilation speed $c_{1}$ the magnitude of which depends on modules $E$ and $G$, Poisson number $\eta$, Lamé constant $\lambda_{1}$ and density $\rho$. Such relations include:

$$
c_{1}=\sqrt{\frac{\lambda_{1}+2 G}{\rho}} ; \lambda_{1}=\frac{E \cdot \eta}{(1+\eta)(1-2 \eta)} .
$$

For $E=2.1 \cdot 10^{11} \mathrm{~Pa}, G=0.8 \cdot 10^{11} \mathrm{~Pa}, \rho=0.795 \mathrm{~N} \cdot \mathrm{s}^{2} \cdot \mathrm{m}^{-4}$, $\eta=0.3$, the value of dilation speed is $c_{1}=5.97 \cdot 10^{3} \mathrm{~m} \cdot \mathrm{s}^{-1}$.

The corresponding dynamic stress in the direction of normal line $x$ is established by the equation:

$$
\sigma_{x}=E \frac{1-\eta}{(1+\eta)(1-2 \eta)} \frac{v_{0}}{c_{1}}=0,47 \cdot 10^{8} v_{0}[\mathrm{~Pa}] .
$$

It is obvious that the dynamic stress value depends on impact speed $v_{0}$ which we will establish on the basis of the following speculation: from the static point of view, the length $L$ of the instant contact spot is non-zero. As a result of rolling away at translational speed $v$, segment length $L$ continuously emerges and disappears in time $t=L \cdot v^{-1}$ which is a very small value even at relatively low translational speeds $v$.

Therefore, when defining speed $v_{0}$ we can start with limit value $\mathrm{L} \rightarrow 0$ which also results in $v_{0} \hat{=} v$. For example, for $v=100 \mathrm{~km} / \mathrm{h}$, we get the stress value of $\sigma_{\mathrm{x}}=1305 \mathrm{MPa}$. We realize that this stress value corresponds to an extremely short instant of time.

The considered medium (steel) can register these short instants. However, this fact is not accepted in classical theories about the limit states of material.

For completeness, let us also state that the components of stress in the plane perpendicular to normal line $x$ are defined by the relation:

$$
\sigma_{y}=\sigma_{z}=\lambda_{1} \frac{v_{1}}{c_{1}} .
$$

In connection with the speculation about the propagation of deformation waves, it is necessary to point out the phenomenon of Rayleigh waves. This wave motion process is specific considering the fact that the plane of oscillation is perpendicular to the tangential plane. The amplitudes in the direction of the normal line decrease exponentially. Therefore, the above described wave motion manifests itself predominantly as skin effect. For details see the research by Brepta (1997) containing the following empirical formula for the speed of propagating these waves:

$$
c_{r}=c_{2}\left(0.153 \eta^{0.89}+0.877\right),
$$

where: speed $c_{2}$ is the speed of transverse wave motion and equals $c_{2}=3.200 \mathrm{~m} \cdot \mathrm{s}^{-1}$. For the Poisson number $\eta=0.3$, we obtain $c_{r}=4.470 \mathrm{~m} \cdot \mathrm{s}^{-1}$. The phenomenon of propagating the described superficial wave motion actually exists. Still, it has not yet been answered which part of energy is transferred by means of this kind of wave motion.

An analogy with high-frequency transfers of electricity applying normal conductors suggests that such skin-effect transfers a considerable part of transported electricity which necessarily brings a question whether it is not the same in case of the dynamic loading of the contact surfaces.

Owing to the fact that thin superficial layers of the rolled-away wheel show substantial changes in material constants, there are conditions for additional waves described by Achenbach (1973). These are shear waves the occurrence of which is conditioned by the existence of a thin elastic layer adhering to an elastic medium with distinctly different material constants.

\section{The Occurrence and Importance of Thermal Effect in the Contact}

Adhesion drive is typical in the occurrence of tangential force $T$ the instant magnitude of which is given by the instant value of normal line wheel force and the status of the contacting surfaces in the contact. The rail vehicle theory uses the slip characteristic, which in principle expresses the tangential deformation property of the surfaces accompanied with increasing tangential slips $s$. The processes taking place in the contact area necessarily have thermodynamic nature and are diffusion processes. In case of small tangential deformations and slips, the generated contact temperature will be very low, so material volumes are in a state near thermodynamic equilibrium. The system behaves as isolated and minimally influenced by any external effects. If the linearity of the dependence of slip $s$ on tangential force $T$ in this area were positively proved (Lata and Čáp 2010), the possibility of changes in the steepness of the slip characteristics could be ruled out. If we accept the established phenomenon of the instant relation between tangential force $T$ and normal line force $R$, i.e. if we introduce the term coefficient of adhesion $\mu$, the slip characteristic is the function of $\mu=f(s)$. For the mean value of the generated contact temperature $T_{K}$ we suggest the following relation:

$$
T_{k}=1.11 \frac{\mu R}{\sqrt[b]{a \lambda \rho \cdot c_{p}}} \Phi,
$$

where: $\mu$ is the coefficient of adhesion; $R$ is wheel force $[\mathrm{N}] ; a, b$ are the lengths of the half-axes of contact surface $[\mathrm{m}] ; \lambda=55.0$ is the thermal conductivity of steel $\left[\mathrm{J} \cdot \mathrm{s}^{-1} \cdot \mathrm{m}^{-1} \cdot \mathrm{K}^{-1}\right] ; \rho=0.79 \cdot 10^{4}$ is the density of steel $\left[\mathrm{N} \cdot \mathrm{s}^{2} \cdot \mathrm{m}^{-4}\right] ; c_{p}=460$ is the specific thermal capacity of steel $\left[\mathrm{J} \cdot \mathrm{kg}{ }^{-1} \cdot \mathrm{K}^{-1}\right], \quad \Phi=\sqrt{v_{1}}-\sqrt{v_{2}}$ is speed function $\left[\left(\mathrm{m} \cdot \mathrm{s}^{-1}\right)^{0.5}\right]$. Then the slip is expressed by the equation:

$$
v_{2}=v_{1}-s \cdot v_{2},
$$


where: $v_{1}$ is the circumferential speed of the wheel and $v_{2}$ is its advancing speed.

The speed function can then be expressed in the following form:

$$
\Phi=\sqrt{v_{1}}-\sqrt{v_{1}-s v_{1}}=(1-\sqrt{1-s}) \sqrt{v_{1}} .
$$

As we are interested in the progress of contact temperatures $T_{K}$ in the entire range of slip $s$, let's introduce the respective analytical function in relation to contact temperature $T_{K}$. Its expression is based on the above mentioned fact that adhesion processes have strong diffusion nature. The processes of a similar type have proved to be successfully described from the probability position, e.g. on the basis of the Gauss distribution. By introducing the error function (Likeš and Machek 1981; Papoulis 1991) expressing the dependence of the coefficient of adhesion $\mu$ on slip $s$, we get the following relation:

$$
\mu(s)=\frac{1}{2} \operatorname{erf}\left(\frac{s}{\sigma \sqrt{2}}\right)=\frac{1}{\sqrt{\pi}} \int_{0}^{\frac{s}{\sigma \sqrt{2}}} \exp \left(-\left(\frac{s^{2}}{2 \sigma^{2}}\right)\right) d s .
$$

Symbol $\sigma$ is the standard deviation of normal distribution (the mean value of the distribution function is zero). The stated function limits to value $\mu=0.5$. According to research done by adhesion drive theoreticians (Lata and Čáp 2010; Spiryagin et al. 2008), adhesion characteristics have a real peak and along with increasing slip s, the adhesion process converts to the process of shear friction. Therefore, we further propose corrected function $\mu(s)=$ cor.erf $(s)$ as a subtraction of two functions while setting the condition that for $s \rightarrow \infty$, the coefficient of adhesion converts into the coefficient of pure shear friction $f$. The corrected function is:

$$
\begin{aligned}
& \mu=\mu_{1}(s)-\mu_{2}(s)=\frac{1}{2} \operatorname{erf}\left(\frac{s}{\sigma_{1} \sqrt{2}}\right)- \\
& a_{1}\left(\operatorname{erf}\left(\frac{s-v_{2}}{\sigma_{2} \sqrt{2}}\right)+1\right) .
\end{aligned}
$$

So as to fulfil the condition stated above, the value of the correction coefficient is given by the equation:

$$
a=0.25-0.5 \cdot f \text {. }
$$

Statistical parameters $\sigma_{1}, \sigma_{2}$ and $v$ determine the shape of the slip characteristics. Their values will be established by experimentation on the upcoming laboratory testing device.

For obtaining essential orientation, Fig. 1 contains the theoretically calculated progress of the slip characteristic for the alternative values of parameter $v_{1}$ and the coefficient of shear friction $f=0.1$.

Progress shown in Fig. 2 indicates that the steepness of the characteristics defined by the tangent in point $[0,0]$ is influenced by parameter $v_{2}$. The actual value of steepness, which from our point of view may be only one, can be determined by experimentation.

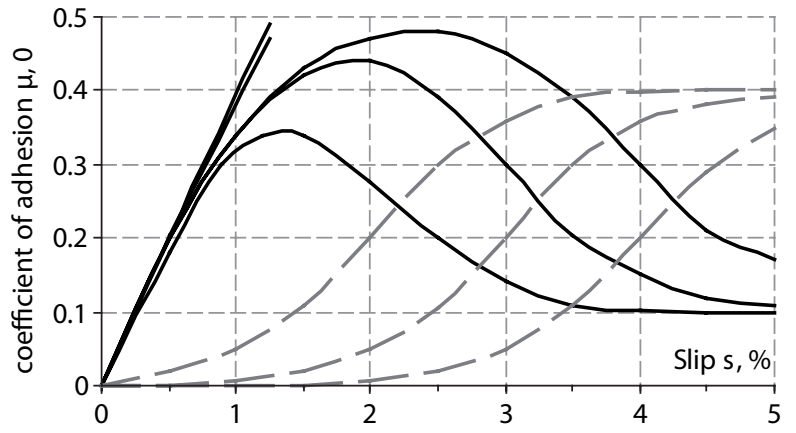

Fig. 1. The dependence of slip characteristics $\mu=f(s)$ on alternative statistical parameters $\sigma_{1}$ (expressed by means of the tangent at the zero point)

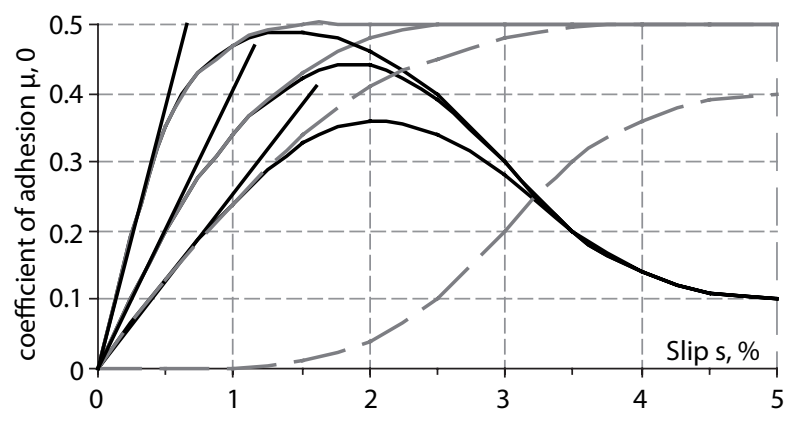

Fig. 2. The dependence of slip characteristics $\mu=f(s)$ on alternative statistical parameters $\sigma_{2}$

So as to set the criterion of linearity, we start with the relation to the derivation of the corrected function at point $[0,0]$ which has the following form:

$$
\begin{aligned}
& \frac{d \mu}{d s}=\frac{1}{\sigma_{1} \sqrt{2 \pi}}-\frac{2 a_{1}}{\sigma_{2} \sqrt{2 \pi}} \exp \left(-\frac{v_{2}^{2}}{2 \sigma_{2}^{2}}\right)= \\
& \frac{1}{\sqrt{2 \pi}}\left(\frac{1}{\sigma_{1}}-\frac{2 a_{1}}{\sigma_{2}} \exp \left(-\frac{v_{2}^{2}}{2 \sigma_{2}^{2}}\right)\right) .
\end{aligned}
$$

For defining the criterion of linearity, the condition that the deviation of function $\mu(s)$ from the tangent at point $[0,0]$ is less than or equal to the deviation was used which corresponds to change in slip by $0.1 \%$ :

$$
\frac{d \mu(0)}{d s} \cdot s=\mu(s+0.1) \text {. }
$$

After substituting the corrected function $\mu(s)=$ cor. $\operatorname{erf}(s)$, we will arrive at the sought dependence of temperature $T_{K}$ on slip $s$ :

$$
T_{K}=\frac{1,11 R}{b} \sqrt{\frac{v_{1}}{a \lambda \rho c_{\mathrm{p}}}}(1-\sqrt{1-s}) \cdot \operatorname{kor} \operatorname{erf}(s) .
$$

The next diagram on Fig. 3 contains the example of the calculated temperatures $T_{K}$ for speeds $v_{1}=10 ; 30$; $50 \mathrm{~m} \cdot \mathrm{s}^{-1}$. Wheel force is $R=10^{5} \mathrm{~N}$, the radius of the wheel is $625 \mathrm{~mm}$, the radius of the profile of the wheel tread is $340 \mathrm{~mm}$ and the radius of the rail head is $300 \mathrm{~mm}$. The corresponding dimensions of the contact spot according to Hertz are $a=14.32 \mathrm{~mm} ; b=5.77 \mathrm{~mm}$. 


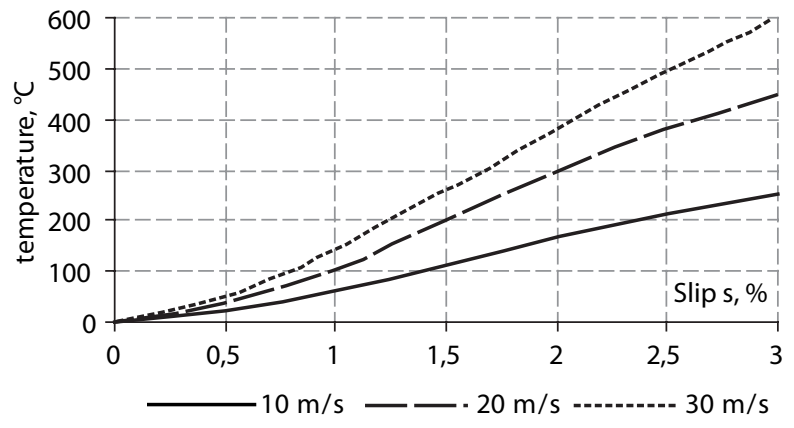

Fig. 3. The dependence of the calculated temperatures $T_{K}$ on speeds $v_{1}=10 ; 30 ; 50 \mathrm{~m} \cdot \mathrm{s}^{-1}$

\section{Laboratory Device for Studying Adhesion Characteristics}

For the experimental study of the progresses of tangential forces at the set or variable slip $s$, another type of a laboratory device was developed, which is expected to verify or supplement the existing opinions based on the established coefficient of adhesion $\mu$, i.e. on the ratio between radial loading force and incurred tangential force. The principle of its activity is described by means of the diagram presented in Fig. 4. The tested samples of material 2, 3 are disc-shaped and have similar diameter.

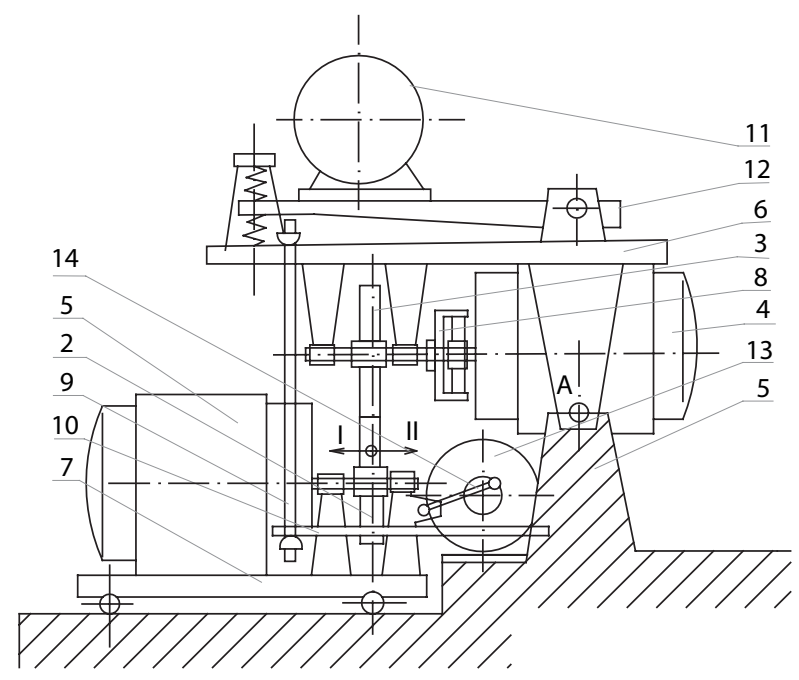

Fig. 4. The testing device for an experimental study on the progress of tangential forces at the set or variable slip $s$

Both discs are driven directly by vector-controlled synchronous servomotors 4,5 . The upper system 3,4 is located on rest 6 pivoted in relation to machine frame 1 around the indicated axis $A$. Servomotor 5 is located on horizontally-sliding rest 7 . The shaft of the upper disc 3 is fitted with torsionally-flexible dynamograph 8 . The radial loading of both discs can be adjusted by means of vertical drawbars 9 linked to girder dynamographs 10 in the bottom part. The dynamic component of this loading is incurred by a pair of rotational vibrators 11 the motors of which are equipped with programmable control.
The bearing of vibrators on spring-loaded rest 12 enables their self-synchronization. The horizontal oscillation of rest 7 (indicated by arrows I, II) bearing servomotor 5 is controlled by program-controlled motor 13 by means of crank mechanism 14.

The device is proposed for two test modes. In the first mode, the speeds of both motors are set so that the selected tangential slip is incurred; it is also possible to set the transverse oscillation of rest 7. Flexible dynamograph 8 sends a signal about the value of the adhesively transferred torque including its changes in time. In this way, it is possible to test working modes within the entire range of the set tangential slips with the contribution of transverse slips. The modes are tested either as stable or transient. The second mode of tests has similar nature, except that one of the servomotors is controlled as a generator. In this case, the slip is incurred by adhesive processes in the contact of both discs.

These tests carefully monitor the dynamic manifests of the system around the maximum of the incurred tangential force where unstable states are presumed on the basis of the classical adhesion theory (Yamazaki et al. 2004; Bureika and Mikaliūnas 2008).

\section{Conclusions}

The paper has presented a new theoretical approach to the loading analysis of contact surfaces during rolling material exploitation. After the statistical calculation of the slip characteristics and their dependences in the introduction, basic information about two new types of testing devices was given for studying the influence of the dynamic load of contact surfaces in the contact area of a rolling kinematical pair. The essential substantiation of building these facilities is presented in the first part of contribution. The next chapter deals with the possible occurrence of wave effects in contact with adhesion drive for a rail vehicle and points out the necessity of experimental research focused on the limit states of the rail and railway wheel material. The final part presents speculation about occurring thermal effects in the contact along slip characteristics described within the entire range on the basis of the probability theory that can be very important and dangerous from the view of material damage in the contact between the rail and railway wheel, for example.

\section{Acknowledgement}

This paper was elaborated with the support of GAČR the performed analyses were financed from the funds of the grant project reg. No. 101/07/0727 named 'Study of Processes at Dynamic Contact of Real Metal Bodies in Relation to Material Changes'.

\section{References}

Achenbach, J. D. 1973. Wave Propagation in Elastic Solids. North-Holland. 425 p.

Beneš, L.; Kaloč, R.; Minář, L. 2008. New approach to the testing of dynamically stressed contact surfaces, Mechanics Transport Communications 3: IX-9-IX-14. 
Beneš, L.; Záhorová, V. 2006. Dynamical loading, thermal effects and temperature generation in the railway wheel / rail contact region, in International Colloquium Advanced Manufacturing and Repair Technologies in Vehicle Industry, 10-12 May 2006, Kollm/Dresden, 119-126.

Brepta, R. 1997. Vlny napětí a rázové jevy v lineárně elastických a viskoelastických prostředích [Strain Waves and Impact Phenomenon in Linear-Elastics and Visco-Elastic Media: Monograph]. LENAM s.r.o, Liberec. 450 p. (in Czech).

Bureika, G.; Mikaliūnas, Š. 2008. Research on the compatibility of the calculation methods of rolling-stock brakes, Transport 23(4): 351-355.

doi:10.3846/1648-4142.2008.23.351-355

Lata, M.; Čáp, J. 2010. The steepness of the rising branch of adhesive characteristics between wheel and rail, Transport 25(1): 17-21. doi:10.3846/transport.2010.03

Likeš, J.; Machek, J. 1981. Počet pravděpodobnosti [Calculus of Probabilities]. SNTL Praha (in Czech).

Papoulis, A. 1991. Probability, Random Variables, and Stochastic Processes. 3rd edition. McGraw-Hill Companies. 666 p.

Spiryagin, M.; Lee, K. S.; Yoo, H. H. 2008. Control system for maximum use of adhesive forces of a railway vehicle in a tractive mode, Mechanical Systems and Signal Processing 22(3): 709-720. doi:10.1016/j.ymssp.2007.09.018

Yamazaki, H.; Nagai, M.; Kamada, T. 2004. A study of adhesion force model for wheel slip prevention control, JSME International Journal Series C - Mechanical Systems Machine Elements and Manufacturing 47(2): 496-501.

doi:10.1299/jsmec.47.496 